\title{
Osmoadaptive Strategies in Halophilic Fungi
}

\author{
Valerie Gonsalves ${ }^{1,2^{*}}$ and Sarita W. Nazareth ${ }^{1}$ \\ ${ }^{\prime}$ Department of Microbiology, Goa University, Taleigao Plateau, Goa, India. \\ ${ }^{2}$ Department of Microbiology, St. Xavier's College, Mapusa, Goa, India \\ "Corresponding authorEmail: valerie.gonsalves@gmail.com \\ (Submitted on November 10, 2020; Accepted on December 20, 2020)
}

\begin{abstract}
Osmoregulatory mechanisms are essential for fungi to survive in hypersaline environments. The osmoregulatory mechanisms, namely 'organic osmolyte' and 'salt-in-cytoplasm' mechanisms were studied in obligate and facultative halophilic fungi isolated from various athalassohaline, thalassohaline and polyhaline econiches, as a response when grown at solar salt concentrations optimal for their growth, as well as one concentration below and one above the optimal. All the halophilic fungi accumulated sugars sucrose and trehalose, and polyols erythritol, ribitol, arabitol, xylitol, inositol, mannitol, and dulcitol at each of these salt concentrations. An increase in the solar salt concentrations in the growth medium resulted in an increase in the total concentration of osmolytes accumulated by the mycelia; a positive correlation was also seen with individual osmolytes. These fungi showed low intracellular accumulation of sodium and potassium cations, which may therefore not contribute significantly in osmoregulation; however, a high concentration of $\mathrm{Na}^{+}$was detected on the fungal cell wall, indicating this to be a protective mechanism for the fungal cell. This indicates that the halophilic fungi examined are essentially osmo-conformers, operating on a mechanism of organic osmolyte accumulation for adaptation to hypersaline environments.
\end{abstract}

Keywords: Compatible solutes, salt-in-cytoplasm, obligate halophilic fungi, facultative halophilic fungi

\section{INTRODUCTION}

In saline environments, microorganisms are surrounded with a low water potential, and hence would tend to lose cytoplasmic water, resulting in cell shrinkage. In order to maintain osmotic equilibrium the cell must decrease the chemical potential of its free water (Kunte et al., 2002). Microbial cells have developed two basic adaptive mechanisms, in order to lower its chemical potential: the 'salt-in-cytoplasm' mechanism and the 'organic osmolyte' or 'compatible solute' mechanism (Kunte et al., 2002).

The key adaptation in archaea is by the 'salt-in-cytoplasm' mechanism (Kunte et al., 2002; Margesin and Schinner, 2001; Roberts, 2005); accumulation of inorganic ions is also reported in Halanaeorobiales, Salinibacter ruber, and Halorhodospira halophila (Harding et al., 2016). Grant (2004) reported that the use of inorganic ions as a mechanism for osmoadaptation is exclusively limited to halophilic prokaryotes. Eukaryotes cannot tolerate high concentrations of intracellular ions (Gunde-Cimerman and Plemenitas, 2006). Fungal cells mainly accumulate compatible solutes to maintain their internal osmotic conditions and keep the intracellular concentrations of sodium ions below the toxic level, while combating hypersaline conditions of the environment (Kogej et al., 2005; Roberts, 2005; GundeCimerman and Plemenitas, 2006; Plemenitas et al., 2008; Kunte et al., 2002; Nazareth et al., 2019). These included a number of polar uncharged solutes. It has been reported that the amount and type of osmolyte varied with the salinity and the growth phase of the fungal culture (Plemenitas et al., 2008), and that physiological responses to increasing salt concentrations vary between the optimal and higher salt concentrations (Perez-Llano et al., 2020).

However, the accumulation of ions for osmoregulation is a less studied mechanism in fungi. Therefore, the present study presents an analysis of the mechanism of osmoregulation by obligate as well as facultative halophilic fungi, with respect to accumulation of compatible solutes as well as of ions, when grown at different salt concentrations.

\section{MATERIALSAND METHODS}

Isolates: Twelve halophilic fungi were selected, previously isolated from different saline environments: the Dead Sea water (DSw) and sediment (DSs) samples (Nazareth et al., 2012), from the estuary of Mandovi, Goa, on the West Coast of the Indian peninsula - from surface and bottom waters $\left(\mathrm{EMw}_{\mathrm{s}}\right.$ and $\left.\mathrm{EMw}_{\mathrm{b}}\right)$ and from sediment (EMs) samples (Gonsalves et al., 2012), from water samples of mangroves at Ribander (MRw) and sediment of solar salterns at Ribander (SRs), Goa (Nayak et al., 2012). The fungi comprised the genera of Aspergillus, Penicillium and Cladosporium, of which some were obligate halophiles: four isolates of Aspergillus penicillioides: DSs40 (GenBank Accession number HQ702385), EM6s 137 (GenBank Accession number JQ240645), EM8 $\mathrm{w}_{\mathrm{s}} 146$ and MRw207, and others were facultative halophiles: A. restrictus DSw14 (GenBank Accession number HQ702382), A. penicillioides EM7 $\mathrm{w}_{\mathrm{s}} 138$, A. versicolor SRs246, A. wentii SRs249, P. corylophilum DSw10, P. waksmanii DSw16, C. Cladosporioides DSw26, C. carpophilum EM9 $\mathrm{w}_{\mathrm{b}} 154$, so classified on the basis of their salt requirement for growth, as recorded earlier (Nazareth et al., 2012; Nayak et al., 2012; Gonsalves et al., 2012).

Analysis of osmolyte accumulation and statistical analysis: The isolates were grown in Czapek Dox Broth supplemented with solar salt at a concentration that supported maximal growth: $10 \%$ for obligate halophiles and $2 \%$ or $5 \%$ or $10 \%$ salt for facultative halophiles, as well as a concentration below and above this optimal concentration. Cultures were harvested in the mid-logarithmic phase and processed by the method as recorded earlier (Nazareth et al., 2019). Sugar and polyol concentrations of the samples were calculated as $\mathrm{mg} \mathrm{g}^{-1}$ wet weight of mycelia.

Pearson correlation coefficients were calculated between the concentration of the total accumulated osmolytes or individual osmolyte and external salinity in the growth medium. Statistical difference was analysed by two-way ANOVA for the effect of different salt concentrations of 
accumulated osmolytes within an isolate, as well as within the genera. Statistical significance was determined at the level of $\mathrm{P}<0.05$.

Analysis of ion concentrations and statistical analysis: Cultures were grown as above, and harvested in the logarithmic phase of growth, briefly rinsed with milliQ water and then suspended in $0.1 \mathrm{~N} \mathrm{HCl}(15 \mathrm{~mL})$ in a flask. This was kept on a shaker at $150 \mathrm{rpm}$ for $15 \mathrm{~min}$ and then filtered through a muslin cloth. The filtrate and washings of the biomass were collected in $\mathrm{HCl}$-treated polypropylene tubes, and used for determination of $\mathrm{Na}^{+}$and $\mathrm{K}^{+}$ions bound to the cell surface. The biomass, after removal of adsorbed ions, was used for extraction of intracellular ions by a modified method of Luard (1982) and of Kelavkar and Chhatpar (1993). The biomass was dried in an oven at $80^{\circ} \mathrm{C}$ to obtain constant dry weight, and then heated in a furnace at $450^{\circ} \mathrm{C}$ for $5 \mathrm{~h}$. The charred biomass was treated with $2 \mathrm{~mL}$ of $3 \mathrm{M} \mathrm{HCl}$ and left overnight for extraction of intracellular ions. MilliQ water, 5 $\mathrm{mL}$, was added to the suspension and filtered through a 0.22 $\mu \mathrm{m}$ Millipore filter into $\mathrm{HCl}-w a s h e d$ polypropylene tubes. The analysis of bound and intracellular $\mathrm{Na}^{+}$and $\mathrm{K}^{+}$ions was done using a flame photometer (ELICO, Model 378), read against the standard curve of $0-100$ ppm of $\mathrm{Na}^{+}$and $\mathrm{K}^{+}$using dilutions of 1000 ppm stock solutions of $\mathrm{NaCl}$ and $\mathrm{KCl}$ prepared in milliQ water.

Pearson correlation coefficient was determined for the amount of $\mathrm{Na}^{+}$and $\mathrm{K}^{+}$ions accumulated intracellularly, and that adsorbed onto the mycelia, with external salt concentration used in the growth medium.

\section{RESULTS}

\section{Sugars and sugar alcohols accumulated by the fungi}

The osmolyte type and concentration in the isolates grown at different concentrations of salt, are depicted in Fig. 1A. All the isolates accumulated intracellular solutes of different lengths ranging from four to six carbon backbone, comprising both sugars and sugar alcohols, albeit in varying concentrations, in response to the different levels of salt supplied in the growth medium,. The sugars included the sugars trehalose and sucrose, the sugar alcohols detected were the four carbon chain erythritol, the five carbon chain ribitol, arabitol, xylitol, and the six carbon chain inositol, mannitol, and dulcitol. The correlation between the accumulation of individual osmolyte, as well as the total pool of osmolytes, and the varied salt levels in the growth medium, are indicated in the following sections.

Obligate halophiles: In the obligate halophilic Aspergillus penicillioides DSs40, EM6s137, EM8w 146 and MRw207, the increase in concentration of salt in the growth medium correlated positively $(r=0.92-0.99)$ with an increase in the concentration of each individual osmolyte, with a few exceptions, namely, a weak correlation in case of ribitol in DSs40, EM6s137 and EM8 $\mathrm{w}_{\mathrm{s}} 146$, inositol in DSs40, and, mannitol and dulcitol in EM6s137. The amount of total osmolytes accumulated in obligate aspergilli ranged from 11$56 \mathrm{mg}$ osmolytes $\mathrm{g}^{-1}$ mycelial wet weight, there being a positive correlation $(\mathrm{r}=0.83-0.99)$ between the total osmolyte pool in the isolates and the salt concentration of the medium.

In each of the isolates EM6 137, EM8ws146 and MRw207, a statistically significant difference $(\mathrm{P}<0.05)$ was seen in the concentration of individual types of osmolytes accumulated in response to increase in salt level in the medium; however, no such difference was seen in isolate DSs 40 .

Facultative halophiles: In all facultative halophilic isolates of aspergilli: $A$. restrictus DSw14, A. penicillioides Em7 $\mathrm{w}_{\mathrm{s}} 138$, A. versicolor SRs246 and $A$. wenti SRs249, penicilli: $P$. corylophilum DSw10 and $P$. waksmanii DSw16; and cladosporia: $C$. cladosporioides DSw26 and $C$. carpophilum $\mathrm{EM} 9 \mathrm{w}_{\mathrm{b}} 154$, the content of individual osmolytes increased with the increase in salt levels in the growth medium, and in most instances displayed a strong positive correlation $(\mathrm{r}=0.88-0.99)$. However, in DSw14 $A$. restrictus, there was a weak positive correlation with mannitol accumulation and a strong negative correlation with sucrose and trehalose accumulation; there was also a weak positive correlation observed in the accumulation of erythritol in P. corylophilum DSw10, and of arabitol and inositol in $\mathrm{EM} \mathrm{w}_{\mathrm{b}} 154 C$. carpophilum. Amongst the facultative halophiles, total osmolytes accumulated g-1 mycelia wet weight was $11-48 \mathrm{mg}$ in aspergilli, 16-63 $\mathrm{mg}$ in penicilli, and $17-50 \mathrm{mg}$ in cladosporia. The total osmolyte concentration increased with an increase in salt concentration in the media $(r=0.87-0.99)$.

Significant difference $(\mathrm{P}<0.5)$ was seen between the increase in salt concentrations in the growth medium and the accumulation of individual osmolytes in all isolates of Aspergillus, Penicillium and of Cladosporium.

\section{Sodium and potassium ions accumulated by the fungi}

The concentrations of $\mathrm{K}^{+}$and $\mathrm{Na}^{+}$cations accumulated and adsorbed by the fungi examined when grown in the presence of different salt concentrations is given in Fig. 1B.

Obligate halophiles: The accumulation of $\mathrm{Na}^{+}$and $\mathrm{K}^{+}$ions in Em8 $\mathrm{w}_{\mathrm{s}} 146$ and MRw207, showed a negative correlation with the increase in salt levels in the medium ( $\mathrm{r}=-0.2$ to -0.6$)$, no correlation in DSw40 $(r=0.06-0.08)$ and a strong positive correlation in EM6s137 $(\mathrm{r}=0.99)$. In all isolates a positive correlation was seen in the amount of $\mathrm{Na}^{+}$ions adsorbed $(\mathrm{r}=$ $0.57-0.96)$, but there was a negative correlation in DSw40 and EM8ws $146(\mathrm{r}=-0.5$ to -.07$)$, or slight positive correlation in EM6s137 and MRw207 ( $\mathrm{r}=0.2)$ with the amounts of $\mathrm{K}^{+}$ ions adsorbed. In all isolates, it was seen that the ratio of intracellular sodium to potassium was maintained to a value of approximately 2.

Facultative halophiles: In the facultative aspergilli, a positive correlation was seen between the increasing levels of salt in the medium and the adsorbed and accumulated $\mathrm{Na}^{+}$and $\mathrm{K}^{+}$ions $(\mathrm{r}=0.2-0.99)$, with the exception of adsorbed $\mathrm{K}^{+}$ions in A. penicillioides $\mathrm{Em} 7 \mathrm{~W}_{\mathrm{s}} 138(\mathrm{r}=-0.48)$ and adsorbed $\mathrm{Na}^{+}$ ion in SRs246 $(r=-0.07)$.

Amongst the penicilli, the increase in salt concentration in the medium showed a positive correlation with the accumulated 


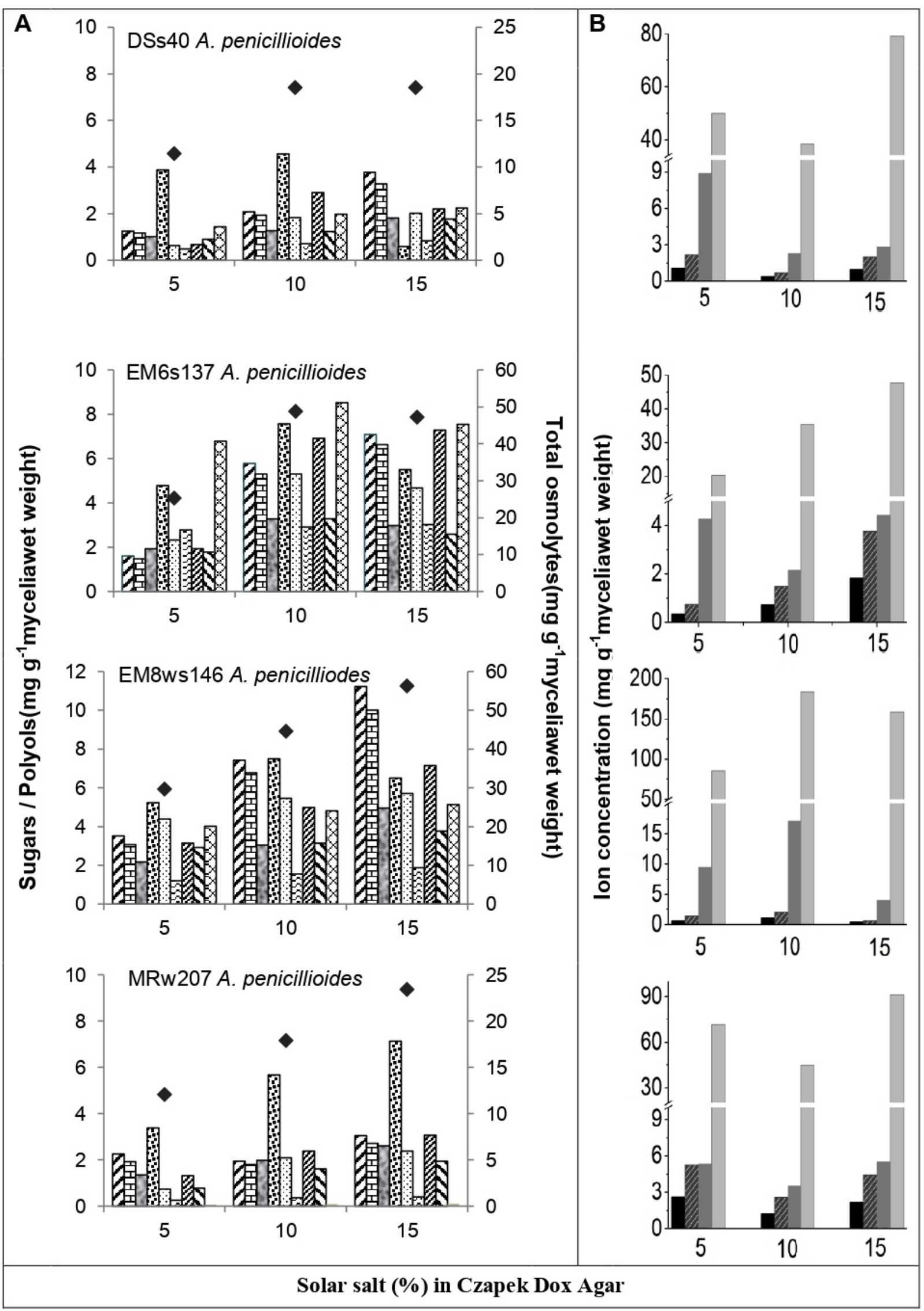




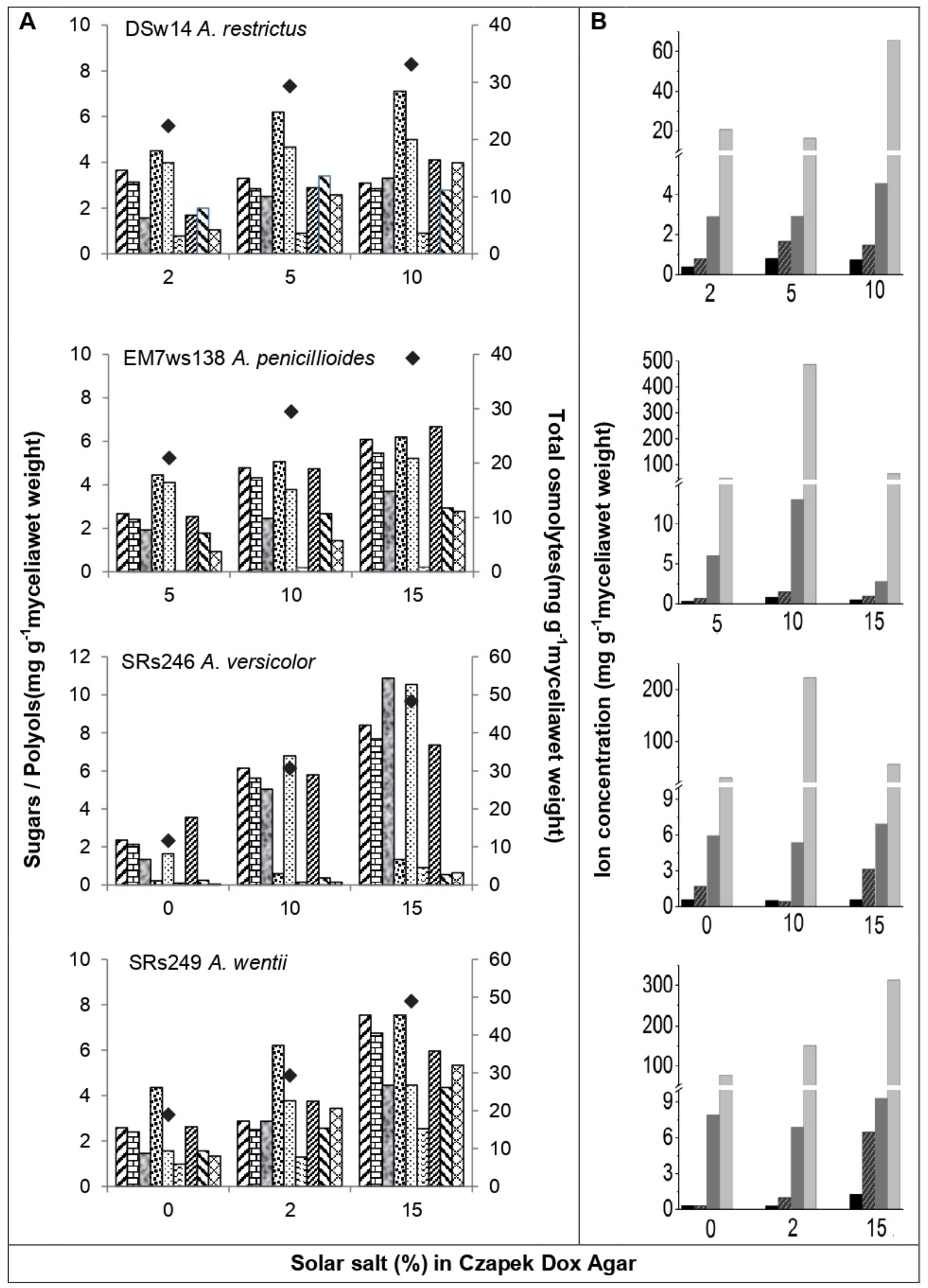




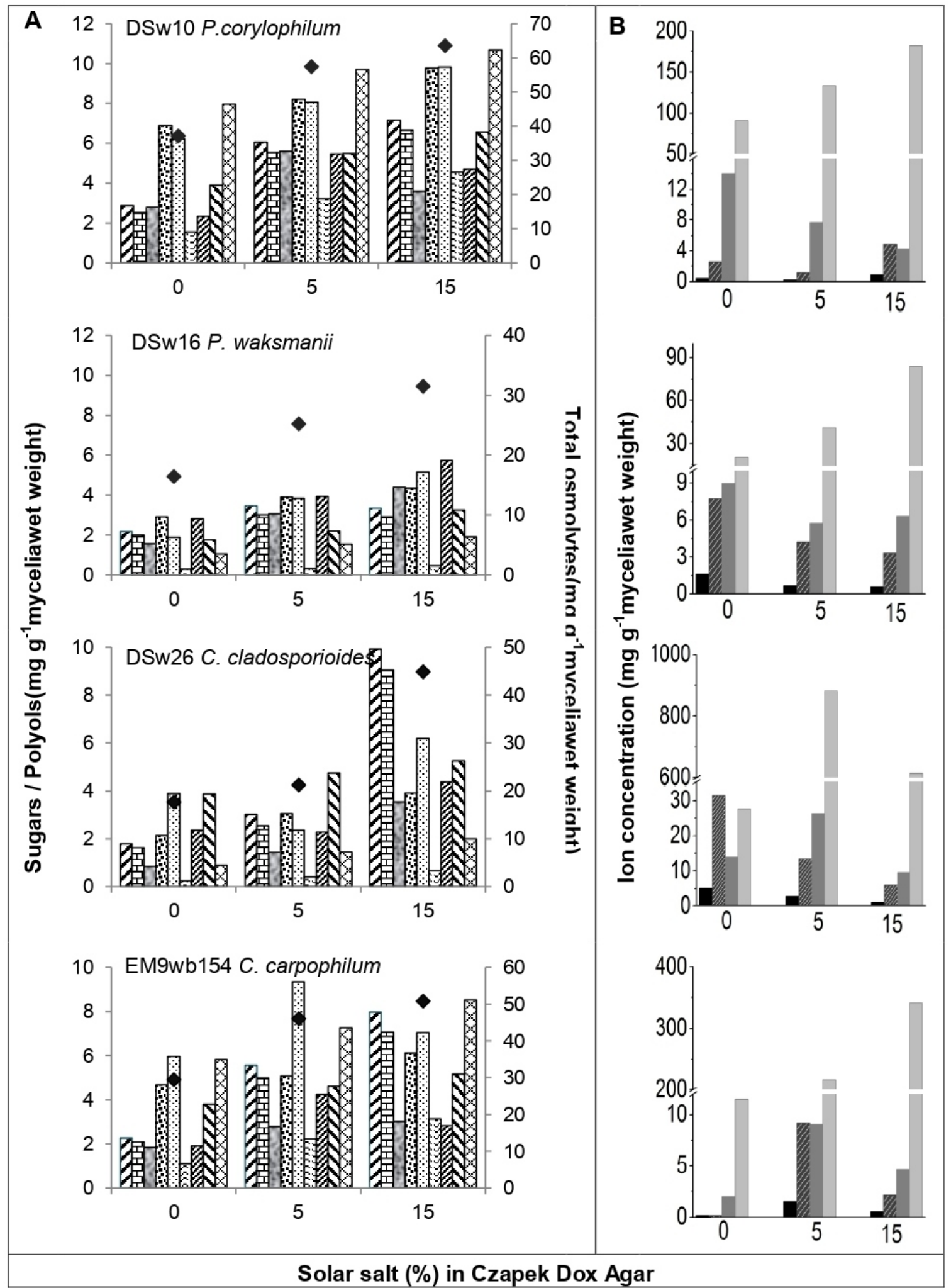

Fig. 1

A Intracellular sugars and polyols in the halophilic isolates: Sucrose $(\mathbb{Z})$, trehalose (圖), erythritol (囦),

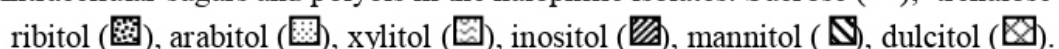

B Accumulated and adsorbed ions in the halophilic isolates: accumulated $\mathrm{K}^{+}(\boldsymbol{\square})$, accumulated $\mathrm{Na}^{+}(\boldsymbol{Z})$, adsorbed $\mathrm{K}^{+}(\square)$, adsorbed $\mathrm{Na}^{+}(\square)$. 
cations in $P$. corylophilum DSw10 $(\mathrm{r}=0.7)$ and a negative correlation in $P$. waksmanii DSw16 $(\mathrm{r}=-0.8)$, while there was with both isolates, a positive correlation with the amount of adsorbed $\mathrm{Na}^{+}(\mathrm{r}=0.9)$ and a negative correlation with adsorbed $\mathrm{K}^{+}$ions $(\mathrm{r}=-0.6$ to -0.9$)$.

Amongst the cladosporia, increase in salt in the growth medium for $C$. cladosporioides DSw26 displayed negative correlation with intracellular $\mathrm{Na}^{+}$and $\mathrm{K}^{+}$ions $(\mathrm{r}=-0.9)$ as well as adsorbed $\mathrm{K}^{+}$ions $(\mathrm{r}=-0.4)$, and a positive correlation with adsorbed $\mathrm{Na}^{+}$ions $(\mathrm{r}=0.5)$; however, in $\mathrm{EM} 9 \mathrm{w}_{\mathrm{b}} 154$, no correlation was seen in intracellular cations $(r=0.02-0.05)$, whereas there was positive correlation with adsorbed cations $(\mathrm{r}=-0.2-0.9)$.

The ratio of intracellular sodium to potassium was nearly constant in cases of the isolates A. penicillioides Em $7 \mathrm{w}_{\mathrm{s}} 138$ and $A$. restrictus DSw14, and was approximately 2 . No such constancy in ratio was seen among the other facultative halophiles belonging to the different genera of Aspergillus, Penicillium and Cladosporium, the ratio being two to three times higher.

\section{DISCUSSION}

Sugars and sugar alcohols accumulated by the fungi: In the present work, the fungi studied accumulated an assortment of osmolytes. The detection of the different osmolytes in response to osmotic stress of sodium chloride, corroborates earlier reports (Beever and Laracy, 1986; Kelavkar and Chhatpar, 1993; Ramirez et al., 2004; Bois et al., 2006; Kogej et al., 2007; Plemenitas et al., 2008; Zajc et al., 2014).

Polyols may be differentially active as compatible solutes, the osmotic pressure they generate being proportionate to their molecular size; however, the larger molecular size polyols may cause a slight inhibition of enzyme activity as compared with those of low molecular weight at equivalent concentrations (Hallsworth and Magan, 1996). Furthermore, it is expected that, as individual compounds are differentially active as compatible solutes, a mixture of solutes would be more efficient in increasing water pressure than a single compound (Ramirez et al., 2004); a mixture would also help to prevent feedback mechanisms caused by high concentration of the product (Kogej et al., 2007), as well as avoid the toxicity associated with high concentration of a single osmolyte on destabilization of protein - protein and DNA - protein interactions (Kogej et al., 2007; Singh et al., 2011; Rydeen et al., 2018).

The facultative halophilic fungal isolates accumulated compatible solutes even in the absence of solar salt added to the medium, indicating alternate roles of these solutes. Indeed, organic osmolytes accumulation is not limited to halophiles but also seen in most organisms as a long-term osmotic balance response (Harding et al., 2016). Polyols are known to serve as carbohydrate reserves, sites for storage of reducing power and translocatory compounds, in coenzyme regulation, as antioxidants and stabilization of proteins (Kelavkar and Chhatpar, 1993; Yancey, 2005; Kogej et al., 2007).
The total osmolyte concentrations in most of the organisms had a sharp linear increase with respect to salt concentration in the external growth medium, irrespective of the decline in growth at salt concentrations higher than that supporting optimal growth, as recorded in our earlier reports (Nazareth et al., 2012; Nayak et al., 2012; Gonsalves et al., 2012); in a few isolates, the total osmolyte concentrations showed a strong increase till the optimal salt concentration, and then a marginal increase thereafter. The osmolytes accumulated would serve the fungi to overcome the osmotic pressure of the external environment; at high external salt concentrations, this would occur at the expense of growth. Thus, a stage may be reached where the internal osmotic pressure may not overcome that of the outside, but the fungus osmoregulates turgour pressure and/or cell volume within limits necessary for growth and survival. At very high concentrations of salt, the cells would use energy for compartmentalization or exclusion of ions, and for synthesis of compatible solutes and proteins that confer salt tolerance, resulting in reduced growth (Redkar et al., 1996)

A wide range of salt tolerance in these fungi, shown in our earlier records (Nazareth et al., 2012; Nayak et al., 2012; Gonsalves et al., 2012) and the ability of many to survive in the absence of salt, may be attributed to the osmolyteaccumulation strategy of these fungi. By this mechanism, fungi can up- or down- regulate the osmolyte concentration according to the salinity of the medium (Kunte et al., 2002; Yancey, 2005).

Sodium and potassium ions accumulated by the fungi: Cells may also increase their internal osmotic pressure by accumulation of inorganic ions like $\mathrm{Na}^{+}$and $\mathrm{K}^{+}$, which is the main osmoregulatory mechanism in archaea (Roberts, 2005).

All isolates accumulated both $\mathrm{Na}^{+}$and $\mathrm{K}^{+}$intracellularly but in low amounts, and thus may be considered as excluders indicating efficient ion flux systems. The same has been reported in the extremely halophilic fungus Wallemia ichthyophaga (Zajc et al., 2014). In the isolates of obligate aspergilli, either a positive, negative or no correlation was seen; however, in all isolates of facultative aspergilli, an isolate of Penicillium and one of Cladosporium, an increase in the salt in the medium caused an increase in the intracellular sodium cation in the medium. At high salinity, there is an increase in the entry of $\mathrm{Na}^{+}$into the cells, causing an overexpression of $\mathrm{Na}^{+}$exporters, thus resulting in maintenance of low $\mathrm{Na}^{+}$concentration inside the cell, in an attempt to reduce the potentially toxic effects of these ions (Gunde-Cimerman and Plemenitas, 2006). Inorganic ions are required for biochemical functions; however once a certain threshold level is reached, a specific ion toxic effect could arise (Bois et al., 2006). Bois et al. (2006) report that cells take in salts as free osmotica; when the accumulated ions reach inhibitory levels and the water stress cannot be compensated for, the cells start using organic compounds as osmotica, at the expense of growth. Thus, the low intracellular accumulation of cations may not contribute significantly to osmoadaptation in fungi in a saline environment.

A high concentration of ion was detected on the cell wall of 
these fungi. In a similar study on the marine fungus Dendryphiella salina, large amounts of sodium and potassium were found in the fungal wall (Clipson and Jennings, 1990). The cell wall is the first defence against the high salinity in the environment. The binding of salt ions on cell wall may contribute to the formation of an external shield that can prevent ions from entering the cell. The biomolecules on fungal cell wall provide negatively charged ligands to which the positive ions of $\mathrm{Na}^{+}$and $\mathrm{K}^{+}$could bind. As with fungal cells, the role of the cell wall of marine fungi in sorption of metal cations has been demonstrated (Bishnoi and Garima, 2005; Gazem and Nazareth, 2012; Bicholkar and Nazareth, 2015). Thus, the $\mathrm{Na}^{+}$and $\mathrm{K}^{+}$cations may also help maintain the integrity of the cell wall as seen in many Haloarchaea (Grant, 2004), the negatively charged glycoproteins of the cell wall requiring stabilization through charge shielding effect and hydrophobic interactions with surrounding cations. It is likely that the inability of obligate fungi to survive in the absence of salt, follows this path more strictly, similar to that in archaea.

The maintenance of a constant internal $\mathrm{Na}^{+} / \mathrm{K}^{+}$ratio at all salt concentrations during growth, by the four obligate halophilic $A$. penicillioides, as well as the facultative halophiles of $A$. penicillioides and of $A$. restrictus, which showed a much delayed growth in absence of added solar salt in the growth medium, indicates that these fungi effectively maintain $\mathrm{Na}^{+}$ and $\mathrm{K}^{+}$homeostasis. The other two facultative halophilic isolates of aspergilli and those of penicilli and cladosporia, which readily grew in absence of added salt, did not show a constant $\mathrm{Na}^{+} / \mathrm{K}^{+}$ratio when grown at the different salt concentration. The ability of the obligate halophiles to maintain constancy in ratio of intracellular $\mathrm{Na}^{+}$to $\mathrm{K}^{+}$indicates their evolutionary adaptation to the environment of high salt concentrations. Neves et al., (1997) also recorded an approximately constant ratio between $\mathrm{K}^{+}$and $\mathrm{Na}^{+}$in halotolerant yeast Debaryomyces hansenii, irrespective of sodium chloride concentrations in the growth medium, and suggest the presence of an efficient transport system capable of maintaining $\mathrm{K}^{+} / \mathrm{Na}^{+}$homeostasis. In any environmental disruption from general conditions, the constituent components of cells coordinate to maintain internal steady state or homeostasis. There is a constant flux of ions in and out of the cells in a controlled fashion with net flux adjusted to accommodate cellular requirements (Niu et al., 1995). In another study, Kogej et al., (2005) reported than in the halophilic isolates of $H$. werneckii the ratio of potassium to sodium was highest when the cells were grown in a medium without added sodium chloride and decreased with increase in concentration of sodium chloride. Similar observations were made in case of the halotolerant $A$. pullulans, the ratio being high when grown in the absence of sodium chloride and decreased when grown at $10 \%$ salt; however, this ratio showed a slight increase at $17 \%$.

\section{CONCLUSION}

From this study, it can be concluded that compatible solute production was the primary mechanism of osmoregulation in both obligate as well as facultative halophilic fungi. These fungi accumulated an assortment of osmolytes and the total concentration of these osmolytes increased with increasing salt concentration in the surrounding medium. The accumulation of only small amounts of $\mathrm{K}^{+}$and $\mathrm{Na}^{+}$is indicative of the exclusion of these ions from the cell, due to their toxicity at higher concentrations. Additionally, the inability of the obligate halophile to grow in the absence of salt, indicates the essentiality of salt in its environment and importance of the adsorbed cations to maintain the integrity of the cell wall for its survival and growth.

\section{ACKNOWLEDGEMENT}

The authors gratefully acknowledge the facilities of Goa University, Goa and National Chemical Laboratory, Pune, during the course of this research work.

\section{REFERENCES}

Beever, R.E. and Laracy, E.P. 1986. Osmotic adjustment in the filamentous fungus Aspergillus nidulans. $J$. Bacteriol. 168:1358-1365.

Bicholkar, A.A and Nazareth S.W. 2015. A comparative study of metal tolerance and sorption capacities of varied fungal genera from metal polluted estuarine environments for potential in metal bioremediation. Kavaka 44:16-29.

Bishnoi, N.R. and Garima N. 2005. Fungus - An alternative for bioremediation of heavy metal containing wastewater: A review. J. Sci. Ind. Res. 64:93-100.

Bois, G., Bertrand, A., Piche, Y., et al. 2006. Growth, compatible solute and salt accumulation of five mycorrhizal fungal species grown over a range of $\mathrm{NaCl}$ concentrations. Mycorrhiza 16: 99-109.

Clipson, N. and Hooley, P. 1995. Salt tolerance strategies in marine fungi. Mycologist 9:3-5.

Gazem, M.A. and Nazareth, S.N. 2012. Sorption of lead and copper from an aqueous phase system by marinederived Aspergillus species. Ann. Microbiol. 65: 503-511.

Gonsalves, V., Nayak, S. and Nazareth, S. 2012. Halophilic fungi in a polyhaline estuarine habitat. J. Yeast Fungal Res. 3: 30-36.

Grant, W.D. 2004. Life at low water activity. Phil. Trans. $R$. Soc. Lond. B. 359: 1249-1267.

Gunde-Cimerman, N. and Plemenitas, A. 2006. Ecology and molecular adaptation of the halophilic black yeast Hortaea werneckii. Rev. Environ. Sci. Biotechnol. 5: 323-331.

Hallsworth, J.E. and Magan, N. 1996. Culture age, temperature, and $\mathrm{pH}$ affect the polyol and trehalose contents of fungal propagules. Appl. Environ. Microb. 62: 2435-2442.

Harding, T, Brown M.W., Simpson. A.G.B. and Roger A.J. 2016. Osmoadaptative strategy and its molecular signature in obligately halophilic heterotrophic protists. Genome Biol. Evol. 8: 2241-2258. 
Kelavkar, U.P. and Chhatpar, H.S. 1993. Polyol concentrations in Aspergillus repens grown under salt stress. World J. Microb. Biot. 9: 579-682.

Kogej, T., Ramos, J., Plemenitas, A. and Gunde-Cimerman, N. 2005. The halophilic fungus Hortaea werneckii and the halotolerant fungus Aureobasidium pullulans maintain low intracellular cation concentrations in hypersaline environments. Appl. Environ. Microbiol. 71: 6600-6605.

Kogej, T., Stein, M., Volkmann, M., et al., 2007. Osmotic adaptation of the halophilic fungus Hortaea werneckii: role of osmolytes and melanization. Microbiol 153: 4261-4273.

Kunte, J.H., Truper, H.G. and Stan-Lotter, S. 2002. Halophilic microorganisms. In: Astrobiology: the Quest for the conditions of life. (Eds.: Horneck, G and Baumstark, C.). Springer, Berlin, pp 185-199.

Luard, E.J. 1982.Accumulation of intracellular solutes by two filamentous fungi in response to growth at low steady state osmotic potential. J. Gen. Microbiol. 128: $2563-2574$.

Margesin, R. and Schinner, F. 2001. Potential of halotolerant and halophilic microorganisms for biotechnology. Extremophiles 5: 73-83.

Nayak, S., Gonsalves, V. and Nazareth, S. 2012. Isolation and salt tolerance of halophilic fungi from mangroves and solar salterns in Goa-India. Indian J. Geo-Mar. Sci. 41: 164-172.

Nazareth, S., Gonsalves, V. and Nayak, S. 2012.A first record of obligate halophilic aspergilli from the Dead Sea. Indian J.Microbiol. 52: 22-27.

Nazareth, S.W., Gonsalves, V. and Gaikwad, S.M. 2019. Compatible solutes in halophilic filamentous fungi. Kavaka 53: 29-33.

Neves, M.L., Oliveira, R.P. and Lucas, C.M. 1997. Metabolic flux response to salt-induced stress in the halotolerant yeast Debaryomyces hansenii. Microbiol. 143: 1133-1139.
Niu, X., Bressan, R.A., Hasegawa, P.M. and Pardo, J. M. 1995. Ion homeostasis in $\mathrm{NaCl}$ stress environments. Plant Physiol. 109: 735-742.

Pérez-Llano, Y., Rodríguez-Pupo, E.C., Druzhinina, I.S., et al. 2020. Stress reshapes the physiological response of halophile fungi to salinity. Cells 9 (3):525.

Plemenitas, A., Vaupotic, T., Lenassi, et al. 2008 Adaptation of extremely halotolerant black yeast Hortaea werneckii to increased osmolarity: a molecular perspective at a glance. Stud. Mycol. 61: 67-75.

Ramirez, M.L., Chulze, S.N. and Magan, N. 2004. Impact of osmotic and matric water stress on germination, growth, mycelia water potentials and endogenous accumulation of sugars and sugar alcohols in Fusarium graminearum. Mycologia 96: 470-478.

Redkar, R.J., Lemke, P.A. and Singh, N.K. 1996. Altered gene expression in Aspergillus nidulians in response to salt stress. Mycologia 88: 256-263.

Roberts, M.F. 2005. Organic compatible solutes of halotolerant and halophilic microorganisms. Saline Systems 1 .

Rydeen A.E., Brustad, E.M. and Pielak, G.J. 2018. Osmolytes and protein-protein interactions. J. Am. Chem. Soc. 140: 7441-7444.

Singh, L.R., Poddar, N.K., Darb, T.A., Kumar, R. and Ahmad, F. 2011. Protein and DNA destabilization by osmolytes: The other side of the coin. Life Sci. 88: 117-125.

Yancey, P.H. 2005. Organic osmolytes as compatible, metabolic and counteracting cytoprotectant in high osmolarity and other stresses. J. Exp. Biol. 208: 2819-2830.

Zajc, J., Kogej, T., Galinski, E.A., et al. 2014. Osmoadaptation strategy of the most halophilic fungus, Wallemia ichthyophaga, growing optimally at salinities above $15 \% \mathrm{NaCl}$. Appl. Environ. Microbiol. 80: 247-256. 